\title{
Serotype-specific invasive disease potential of Streptococcus pneumoniae in Czech children
}

Correspondence

Helena Zemlickova

hzemlickova@szu.cz

Received 18 December 2009

Accepted 21 May 2010

\author{
Helena Zemlickova, Vladislav Jakubu, Pavla Urbaskova, Jitka Motlova, \\ Martin Musilek and Vaclava Adamkova
}

National Institute of Public Health, Šrobárova 48, 10042 Prague 10, Czech Republic

\section{INTRODUCTION}

Streptococcus pneumoniae is a major cause of invasive disease in children and adults worldwide (Marrie, 2000; Hausdorff et al., 2000). The pneumococcal outer surface is covered by an immunogenic polysaccharide capsule which forms the most important virulence factor since it protects pneumococci from phagocytosis (Watson \& Musher, 1999). Capsular polysaccharides are highly heterogeneous. To date, 92 pneumococcal serotypes have been identified (Bratcher et al., 2009), although only approximately 10 of them are commonly the cause of invasive disease (Hausdorff et al., 2000). The major ecological niche of pneumococci is the nasopharynx of young children. Considering the extent of exposure to pneumococci, the invasive disease is rare with a number of virulence factors influencing the success of particular serotypes (Hava et al., 2003). Recently, attention has been devoted to the prevention of pneumococcal infection via a suitable immunization strategy. The use of a 7-valent pneumococcal conjugate vaccine (PCV7) covering serotypes $4,6 \mathrm{~B}, 9 \mathrm{~V}$, $14,18 \mathrm{C}, 19 \mathrm{~F}$ and $23 \mathrm{~F}$ has reduced the incidence of invasive pneumococcal disease in vaccinated children as well as in the non-vaccinated herd immunity-protected population (Center, 2007). However, alarming reports emerged of a partial replacement of invasive strains with non-vaccine serotypes in vivo (Hanage, 2008). Since the vaccine only protects against a limited number of serotypes, it is essential to determine the ability of various serotypes and clones to cause the invasive disease. Vaccine selective

Abbreviations: MLST, multilocus sequence typing; OR, odds ratio; PCV7, 7-valent pneumococcal conjugate vaccine; ST, sequence type.

A figure showing the results of the eBURST analysis is available with the online version of this paper. pressure might promote the occurrence of minor but invasive serotypes or the reappearance of vaccine serotypes which have acquired a non-vaccine serotype capsule (Brueggemann et al., 2007). Epidemiological studies have been conducted which compared the prevalence of serotypes among invasive and carriage isolates within the population of a geographical area (Takala et al., 1996; Brueggemann et al., 2003; Sandgren et al., 2004; Hanage et al., 2005) in order to better understand the invasiveness of pneumococci. PCV7 has been available in the Czech Republic since September 2005. The vaccination is administered on parental request and is paid for by parents; PCV7 coverage in children is reported to be $66 \%$ (Motlova et al., 2009). Since January 2009, PCV7 has been approved for use with children exhibiting a higher risk of pneumococcal disease and is covered by health insurance.

Multilocus sequence typing (MLST) allows the precise description and identification of particular clones occurring in the invasive disease and carriage and detects the dissemination of a genotype within different serotypes (McGee et al., 2001; Enright \& Spratt, 1998). The purpose of this study was to determine the serotype-specific invasive disease potential of pneumococci isolated from children under 6 years of age in the Czech Republic before the widespread use of pneumococcal conjugate vaccines.

\section{METHODS}

Isolates. All the available pneumococcal invasive isolates $(n=138)$ collected from children under 6 years of age and referred to the National Institute of Public Health in the pre-vaccine period of 19962004 were studied. Non-duplicate isolates cultured from blood $(n=74)$ and cerebrospinal fluid $(n=64)$ were provided by 54 microbiology laboratories located throughout the Czech Republic 
and covering $\sim 85 \%$ of the population. Carriage isolates $(n=153)$ collected during the winter of 2004-2005 from the nasopharyngeal swabs of 3-5-year-olds attending 16 day-care centres in nine cities were used for comparison purposes (Zemlickova et al., 2006). Isolates were serotyped using the Quellung reaction employing type and factor sera provided by the Statens Serum Institut, Copenhagen, Denmark (Sorensen, 1993).

Antibiotic susceptibility testing. MICs of penicillin, erythromycin, chloramphenicol, tetracycline and trimethoprim-sulfamethoxazole were determined using the broth microdilution method as described by the Clinical and Laboratory Standards Institute (CLSI) (CLSI, 2009). CLSI interpretation criteria were used to read the results, except for tetracycline and erythromycin where CLSI intermediate values were considered to be resistance (CLSI, 2009). The interpretation of penicillin MICs followed the CLSI criteria for oral penicillin.

MLST. The molecular typing of invasive isolates $(n=112)$ was provided for only the 11 most abundant serotypes. With regard to carriage isolates $(n=60)$, typing was performed on those serotypes showing a significant association with either the invasive disease (serotypes $9 \mathrm{~V}$ and 14) or carriage (serotypes 6A and 23F). The seven housekeeping gene internal fragment sequences were determined using PCR employing the primers described by Enright \& Spratt (1998). Alleles and sequence types (STs) were assigned using data on S. pneumoniae available on the MLST website (www.mlst.net). New alleles and new ST allelic profiles were subsequently submitted electronically to the $S$. pneumoniae MLST database. ST analysis was performed by means of the eBURST program (Feil et al., 2004).

Statistical analysis. Empirical odds ratios (ORs) and $95 \%$ confidence intervals, used to estimate invasiveness for individual serotypes, were calculated using EpiInfo software (version 3.4) as previously described (Brueggemann et al., 2003). An OR $>1$ indicated a higher probability of a serotype causing the invasive disease, while an OR $<1$ was considered to be associated with carriage. Since the number of paediatric invasive isolates per year is low ( 15 isolates), the entire collection of strains from the period 1996-2004 was used for calculation purposes even though the invasive and carriage isolate collection years do not completely overlap. The robustness of ORs calculated for the entire invasive isolate dataset was tested against the invasive isolate subset $(n=49)$ not including isolates from children younger than 3 years. The recalculated ORs revealed the same associations between both the invasive disease and carriage and serotypes; statistical efficiency was reduced, however, due to the low number of isolates. Since the same results were obtained for both the full dataset and the subset, the whole invasive isolate collection was included for the calculation of ORs.

\section{RESULTS AND DISCUSSION}

A total of 138 invasive pneumococci from children under 6 years of age, including $89(64.5 \%)$ isolates recovered from the youngest children sampled $(<2$ years of age), were collected during the period 1996-2004 throughout the Czech Republic. Twenty-five different serotypes were identified only 11 of which were represented by at least five isolates (Table 1). The most common invasive serotypes proved to be $6 \mathrm{~B}, 14,23 \mathrm{~F}, 19 \mathrm{~F}, 9 \mathrm{~V}, 18 \mathrm{C}, 1$ and 6A. The estimated coverage rates of the 7- (PCV7), 10- and 13 -valent pneumococcal conjugate vaccines were $65.2 \%$, $76.8 \%$ and $88.4 \%$, respectively. Serotypes $23 \mathrm{~F}, 6 \mathrm{~A}, 6 \mathrm{~B}, 19 \mathrm{~F}$, $15 \mathrm{~B}$ and $18 \mathrm{C}$ were the most predominant in the carriage strain set $(n=153)$. Serotype 1 was common in the invasive
Table 1. Statistical differences in serotype distribution of pneumococcal isolates $(n=291)$

Serotypes rank ordered by number of invasive isolates.

\begin{tabular}{|c|c|c|c|c|c|}
\hline \multirow[t]{2}{*}{ Serotype } & \multicolumn{3}{|c|}{ No. of isolates } & \multirow[t]{2}{*}{ OR $(95 \% \mathrm{CI})^{\star}$} & \multirow[t]{2}{*}{$P$} \\
\hline & $n$ & Invasive & Carriage & & \\
\hline $6 \mathrm{~B}$ & 44 & 23 & 21 & $1.3(0.6-2.5)$ & \\
\hline 14 & 24 & 19 & 5 & $4.7(1.6-14.9)$ & 0.001 \\
\hline $23 \mathrm{~F}$ & 43 & 13 & 30 & $0.4(0.2-0.9)$ & 0.014 \\
\hline $19 \mathrm{~F}$ & 22 & 12 & 10 & $1.4(0.5-3.5)$ & \\
\hline $9 \mathrm{~V}$ & 10 & 9 & 1 & $10.6(1.3-226.4)$ & 0.006 \\
\hline $18 \mathrm{C}$ & 18 & 9 & 9 & $1.1(0.4-3.2)$ & \\
\hline 1 & 9 & 9 & 0 & - & \\
\hline $6 \mathrm{~A}$ & 32 & 8 & 24 & $0.3(0.1-0.8)$ & 0.007 \\
\hline $7 \mathrm{~F}$ & 7 & 6 & 1 & $6.9(0.8-154.2)$ & \\
\hline 3 & 11 & 5 & 6 & $0.9(0.2-3.5)$ & \\
\hline 4 & 7 & 5 & 2 & $2.8(0.5-21.5)$ & \\
\hline $10 \mathrm{~A}$ & 10 & 4 & 6 & $0.7(0.2-3.0)$ & \\
\hline $9 \mathrm{~N}$ & 3 & 2 & 1 & $2.2(0.2-63.0)$ & \\
\hline $19 \mathrm{~A}$ & 2 & 2 & 0 & - & \\
\hline $28 \mathrm{~F}$ & 2 & 2 & 0 & - & \\
\hline 8 & 2 & 1 & 1 & $1.1(0-41.0)$ & \\
\hline 38 & 2 & 1 & 1 & $1.1(0-41.0)$ & \\
\hline 37 & 3 & 1 & 2 & $0.5(0.02-7.8)$ & \\
\hline $23 \mathrm{~A}$ & 4 & 1 & 3 & $0.4(0.01-4.0)$ & \\
\hline $11 \mathrm{~A}$ & 9 & 1 & 8 & $0.1(0.01-1.1)$ & \\
\hline 5 & 1 & 1 & 0 & - & \\
\hline $12 \mathrm{~F}$ & 1 & 1 & 0 & - & \\
\hline $17 \mathrm{~F}$ & 1 & 1 & 0 & - & \\
\hline $33 \mathrm{~F}$ & 1 & 1 & 0 & - & \\
\hline 40 & 1 & 1 & 0 & - & \\
\hline 15B & 10 & 0 & 10 & - & \\
\hline $15 \mathrm{C}$ & 7 & 0 & 7 & - & \\
\hline $35 \mathrm{~F}$ & 5 & 0 & 5 & - & \\
\hline Total & 291 & 138 & 153 & & \\
\hline
\end{tabular}

${ }^{\star} \mathrm{CI}$, Confidence interval.

isolates; no serotype 1 isolate was found amongst the carriage strains.

To estimate the invasive disease potential of the serotypes, the authors compared the invasive isolates with a set of nasopharyngeal isolates collected from children under 6 years of age. Serotype ORs are shown in Table 1. The highest ORs were exhibited by serotypes $9 \mathrm{~V}, 7 \mathrm{~F}, 14$ and 4 , although only serotypes $9 \mathrm{~V}$ and 14 showed a significant association with the invasive disease. Conversely, serotypes $6 \mathrm{~A}$ and $23 \mathrm{~F}$ were found to be significantly associated with carriage.

One hundred and twelve (95\%) viable isolates of the most prevalent invasive serotypes, i.e. $6 \mathrm{~B}, 14,23 \mathrm{~F}, 19 \mathrm{~F}, 9 \mathrm{~V}, 18 \mathrm{C}, 1$, $6 \mathrm{~A}, 7 \mathrm{~F}, 3$ and 4, were analysed using MLST. A total of 59 unique STs were identified, 21 of which held new alleles or novel combinations of previously identified alleles (Table 2). With regard to serotypes $6 \mathrm{~A}, 9 \mathrm{~V}, 14$ and 23F, MLST revealed 
Table 2. Sequence types determined in pneumococcal isolates from invasive disease and carriage in the Czech Republic

New STs and new alleles are shown in bold.

\begin{tabular}{|c|c|c|c|c|c|c|c|c|c|c|c|c|c|c|}
\hline Serotype & $\begin{array}{l}\text { Total no. of } \\
\text { isolates within } \\
\text { serotype }\end{array}$ & ST & aroE & $g d h$ & $g k i$ & rec & spi & $x p t$ & $d d l$ & $\begin{array}{c}\text { Antibiotic } \\
\text { susceptibility } \\
\text { profile }^{\star}\end{array}$ & Invasive & Carriage & $\begin{array}{l}\text { Total no. } \\
\text { of isolates } \\
\text { within ST }\end{array}$ & Comments \\
\hline \multirow[t]{3}{*}{3} & 4 & 180 & 7 & 15 & 2 & 10 & 6 & 1 & 22 & & 1 & & 1 & \\
\hline & & 232 & 13 & 9 & 15 & 14 & 10 & 16 & 1 & & 1 & & 1 & \\
\hline & & 378 & 13 & 9 & 15 & 14 & 10 & 16 & 19 & & 2 & & 2 & \\
\hline \multirow{3}{*}{4} & & 1022 & 8 & 8 & 4 & 1 & 15 & 1 & 20 & TET R & 1 & & 1 & \\
\hline & & 205 & 10 & 5 & 4 & 5 & 13 & 10 & 18 & & 2 & & 2 & Sweden $^{4}-38$ \\
\hline & & 247 & 16 & 13 & 4 & 5 & 6 & 10 & 14 & & 1 & & 1 & \\
\hline \multirow[t]{3}{*}{14} & 24 & 15 & 1 & 5 & 4 & 5 & 5 & 3 & 8 & CMP R, SXT R & 1 & & 1 & \\
\hline & & 124 & 7 & 5 & 1 & 8 & 14 & 11 & 14 & SXT R(1) & 17 & 5 & 22 & Netherlands ${ }^{14}-35$ \\
\hline & & 143 & 7 & 5 & 10 & 18 & 6 & 8 & 1 & PEN I, TET R, ERY R & 1 & & 1 & \\
\hline $18 \mathrm{C}$ & & 193 & 8 & 10 & 2 & 16 & 1 & 26 & 1 & & 1 & & 1 & \\
\hline \multirow[t]{6}{*}{$19 \mathrm{~F}$} & 12 & 423 & 1 & 5 & 4 & 12 & 5 & 3 & 8 & TET R(5), CMP R(5) & 6 & & 6 & \\
\hline & & 1815 & 1 & 5 & 4 & 12 & 5 & 3 & 159 & TET R, CMP R & 1 & & 1 & \\
\hline & & 380 & 7 & 11 & 19 & 1 & 6 & 20 & 1 & SXT R(2) & 2 & & 2 & \\
\hline & & 2845 & 8 & 10 & 2 & 5 & 9 & 48 & 264 & & 1 & & 1 & \\
\hline & & 2027 & 16 & 127 & 9 & 1 & 1 & 187 & 9 & & 1 & & 1 & \\
\hline & & 251 & 18 & 2 & 22 & 1 & 9 & 23 & 14 & SXT R & 1 & & 1 & \\
\hline \multirow[t]{4}{*}{$23 \mathrm{~F}$} & 43 & 36 & 1 & 8 & 4 & 1 & 1 & 4 & 6 & TET R(1), CMP R(1) & 6 & 22 & 28 & \\
\hline & & 1064 & 1 & 8 & 4 & 1 & 1 & 4 & 14 & & 1 & & 1 & \\
\hline & & 2802 & 1 & 8 & 4 & 1 & 1 & 11 & 6 & & & 2 & 2 & \\
\hline & & 625 & 1 & 8 & 4 & 1 & 6 & 4 & 6 & $\begin{array}{l}\text { PEN I, TET R, ERY } \\
\text { R, CMP R, SXT R }\end{array}$ & 1 & & 1 & \\
\hline \multirow[t]{14}{*}{$6 \mathrm{~A}$} & 31 & 395 & 1 & 5 & 7 & 12 & 17 & 1 & 14 & & & 1 & 1 & \\
\hline & & 600 & 5 & 10 & 9 & 43 & 13 & 1 & 14 & & 1 & 2 & 3 & \\
\hline & & 1143 & 7 & 25 & 4 & 1 & 15 & 1 & 28 & & & 3 & 3 & \\
\hline & & 2804 & 7 & 25 & 4 & 4 & 6 & 20 & 8 & & & 1 & 1 & \\
\hline & & 1769 & 7 & 25 & 4 & 4 & 6 & 20 & 28 & & 1 & 1 & 2 & \\
\hline & & 2847 & 7 & 25 & 4 & 4 & 19 & 20 & 28 & & & 2 & 2 & \\
\hline & & 2844 & 7 & 25 & 9 & 1 & 6 & 14 & 263 & PEN I(1) & 2 & 1 & 3 & \\
\hline & & 2806 & 2 & 13 & 1 & 1 & 6 & 19 & 8 & PEN I(2) & & 2 & 2 & \\
\hline & & 2805 & 2 & 13 & 164 & 1 & 6 & 19 & 14 & & 1 & 8 & 9 & \\
\hline & & 2846 & 2 & 13 & 9 & 1 & 6 & 14 & 14 & & 1 & & 1 & \\
\hline & & 2861 & 107 & 13 & 9 & 1 & 6 & 19 & 14 & & 1 & & 1 & \\
\hline & & 2848 & 8 & 10 & 2 & 16 & 1 & 195 & 1 & & & 1 & 1 & \\
\hline & & 2849 & 8 & 10 & 15 & 1 & 19 & 88 & 1 & & & 1 & 1 & \\
\hline & & 2850 & 8 & 60 & 15 & 1 & 1 & 28 & 14 & & & 1 & 1 & \\
\hline \multirow[t]{4}{*}{$6 \mathrm{~B}$} & 21 & 1784 & 2 & 13 & 9 & 1 & 6 & 19 & 8 & & 1 & & 1 & \\
\hline & & 273 & 5 & 6 & 1 & 2 & 6 & 1 & 14 & TET R, ERY R & 1 & & 1 & Greece $^{6 \mathrm{~B}}-22$ \\
\hline & & 1782 & 5 & 10 & 1 & 43 & 13 & 1 & 7 & & 1 & & 1 & \\
\hline & & 193 & 8 & 10 & 2 & 16 & 1 & 26 & 1 & & 1 & & 1 & \\
\hline
\end{tabular}


Table 2. cont.

\begin{tabular}{|c|c|c|c|c|c|c|c|c|c|c|c|c|c|c|}
\hline Serotype & $\begin{array}{l}\text { Total no. of } \\
\text { isolates within } \\
\text { serotype }\end{array}$ & ST & aroE & $g d h$ & $g k i$ & rec & $s p i$ & $x p t$ & $d d l$ & $\begin{array}{c}\text { Antibiotic } \\
\text { susceptibility } \\
\text { profile }^{\star}\end{array}$ & Invasive & Carriage & $\begin{array}{c}\text { Total no. } \\
\text { of isolates } \\
\text { within ST }\end{array}$ & Comments \\
\hline & & 315 & 20 & 28 & 1 & 1 & 15 & 14 & 14 & $\begin{array}{l}\text { TET R(2), ERY } \\
\text { R(2), SXT R(1) }\end{array}$ & 2 & & 2 & Poland $^{6 \mathrm{~B}}-20$ \\
\hline & & 1125 & 7 & 5 & 1 & 2 & 6 & 1 & 138 & PEN I, ERY R, SXT R & 1 & & 1 & \\
\hline & & 145 & 7 & 6 & 1 & 1 & 6 & 15 & 14 & SXT R & 1 & & 1 & \\
\hline & & 176 & 7 & 13 & 8 & 6 & 10 & 6 & 14 & SXT R(1) & 6 & & 6 & \\
\hline & & 1770 & 7 & 13 & 41 & 6 & 10 & 6 & 14 & & 5 & & 5 & \\
\hline & & 1814 & 58 & 28 & 1 & 1 & 15 & 14 & 136 & SXT R & 1 & & 1 & \\
\hline & & 1783 & 58 & 28 & 1 & 1 & 17 & 14 & 136 & SXT R & 1 & & 1 & \\
\hline $7 F$ & 5 & 191 & 8 & 9 & 2 & 1 & 6 & 1 & 17 & & 5 & & 5 & Netherlands ${ }^{7 \mathrm{~F}}-39$ \\
\hline \multirow[t]{2}{*}{$9 \mathrm{~V}$} & 10 & 156 & 7 & 11 & 10 & 1 & 6 & 8 & 1 & $\begin{array}{l}\text { PEN I(4), ERY R(2), } \\
\text { SXT R(4) }\end{array}$ & 4 & & 4 & Spain $^{9 \mathrm{~V}}-3$ \\
\hline & & 162 & 7 & 11 & 10 & 1 & 6 & 8 & 14 & SXT R(1) & 5 & 1 & 6 & \\
\hline Total & 172 & & & & & & & & & & 112 & 60 & 172 & \\
\hline
\end{tabular}

${ }^{\star}$ PEN, Penicillin; CMP, chloramphenicol; SXT, trimethoprim-sulfamethoxazole; ERY, erythromycin; TET, tetracycline; I, intermediate resistant (MIC of PEN $0.12 \leqslant \mathrm{MIC}<1 \mu \mathrm{g} \mathrm{ml}^{-1}$ ); R, resistant; in parentheses is shown the number of non-susceptible isolates.

8 exclusively invasive disease STs, 11 exclusively carriage STs and 9 combined invasive disease and carriage STs. Overall, ST diversity was higher with regard to serotypes showing no affinity either to cause invasive disease or to colonize the nasopharynx and was even more remarkable in serotypes exhibiting reduced invasive disease potential $(\mathrm{OR}<1)$. One dominant clone was found in each of the $9 \mathrm{~V}, 7 \mathrm{~F}, 14$ and 1 serotypes, thus displaying high invasive disease potential; conversely, no clonality was determined for serotype 4 (OR $2.8)$. The ST36 (65.1\%) serotype was the most prevalent of the $23 \mathrm{~F}$ isolates, showing a significantly lower invasive disease potential. The results of eBURST analysis are shown in Supplementary Fig. S1 in JMM Online.

Both the major virulence factor of $S$. pneumoniae in the polysaccharide capsule and the prominence of certain serotypes in paediatric invasive disease are well known (Brueggemann et al., 2004). Nevertheless, the general assumption that the rank order of serotypes causing invasive pneumococcal disease reflects the level of their invasive disease potential would appear to be incorrect. Recent studies have revealed that certain serotypes that are highly prevalent in the invasive disease may have a lower invasive disease potential than other minor serotypes (Brueggemann et al., 2003; Sandgren et al., 2004). A comparison of serotype distribution among carriage and invasive isolates from children from the same community suggests that certain serotypes have a higher chance of causing the invasive disease due to the high level of exposure to such serotypes due to their being frequent colonizers (Brueggemann et al., 2003). This fact is significant since the benefits of conjugate vaccine could be rendered less efficacious by an increase in non-vaccine serotypes in the invasive disease. Moreover, the findings would seem to suggest it most likely that each serotype has specific biological characteristics that determine its ability to cause the invasive disease or colonize the nasopharynx.

Since pneumococcal meningitis and bacteraemia are relatively rare events and serotype distribution varies slightly between countries, the repetition of such studies might be helpful in strengthening previous results as well as in confirming the general relevance of the conclusions. The results of this study were consistent with the findings of similar studies from the UK (Brueggemann et al., 2003), Sweden (Sandgren et al., 2004) and Finland (Hanage et al., 2005). Although serotype 18C did not exhibit the strong association with the invasive disease reported by the aforementioned studies, Czech serotypes 4, 7F, 9V and 14 similarly exhibited ORs $>1$ and serotype 1 was only recovered in invasive isolates. Analogous patterns were observed for serotypes $6 \mathrm{~A}$ and $23 \mathrm{~F}$, which prevailed in the carriage isolates of all the datasets. The fact that the carriage sampling period was not completely contiguous with that of the disease might be considered a limitation of the study. However, similarly consistent results with regard to serotype-specific OR estimates were found in the Finnish study (Hanage et al., 2005) using the same sampling frame approach, and in the Swedish study, which compared the invasive isolates of all age groups with those carried by children (Sandgren et al., 2004).

Three invasive clones, i.e. Netherlands ${ }^{7 \mathrm{~F}}-39$, Spain $^{9 \mathrm{~V}}-3$ and Netherlands ${ }^{14}-35$, were found to be predominant in this study as well as in those from Oxford, Finland and Sweden. The most obvious differences between these three studies and the Czech study concerned the estimated OR of serotype $18 \mathrm{C}$, which exhibited low potential for invasion in this study, and the lower than previously detected proportion of Sweden ${ }^{4}-38$ clone (ST205) in Czech serotype 4 isolates (Brueggemann et al., 2003; Sandgren et al., 2004; 
Hanage et al., 2005). Since seven serotype 18C carriage isolates were collected in five different day-care centres, it can be concluded that their presence in the carriers is not the result of the spread of a particular clone in a closed environment. Moreover, MLST performed on invasive isolates identified five different STs which were not related to STs exhibiting high ORs in the Oxford (ST113) or Finnish (ST496) studies.

This study demonstrates associations between invasive disease and particular pneumococcal serotypes and clones. Serotype 9V, 7F, 14 and 1 isolates caused invasive disease far more frequently than the other serotypes studied. Following the introduction of the pneumococcal vaccine in routine immunization programmes, the repetition of such studies is highly recommended in order to assess the impact of the vaccine on the pneumococcal population.

\section{ACKNOWLEDGEMENTS}

We would like to thank the regional microbiology laboratories for providing pneumococcal isolates. This study was partly supported by research grant A/CZ0046/2/0007 from Iceland, Liechtenstein and Norway via the EEA financial mechanism and research grant IGA 9643-4 from the Internal Grant Agency, Czech Ministry of Health. This publication made use of the Multi Locus Sequence Typing website (http://www.mlst.net) at Imperial College London developed by David Aanensen and funded by the Wellcome Trust.

\section{REFERENCES}

Bratcher, P. E., Park, I. H., Hollingshead, S. K. \& Nahm, M. H. (2009). Production of a unique pneumococcal capsule serotype belonging to serogroup 6. Microbiology 155, 576-583.

Brueggemann, A. B., Griffiths, D. T., Meats, E., Peto, T., Crook, D. W. \& Spratt, B. G. (2003). Clonal relationships between invasive and carriage Streptococcus pneumoniae and serotype- and clone-specific differences in invasive disease potential. J Infect Dis 187, 1424-1432.

Brueggemann, A. B., Peto, T. E., Crook, D. W., Butler, J. C., Kristinsson, K. G. \& Spratt, B. G. (2004). Temporal and geographic stability of the serogroup-specific invasive disease potential of Streptococcus pneumoniae in children. J Infect Dis 190, 1203-1211.

Brueggemann, A. B., Pai, R., Crook, D. W. \& Beall, B. (2007). Vaccine escape recombinants emerge after pneumococcal vaccination in the United States. PLoS Pathog 3, el68.

Center, K. J. (2007). Prevenar ${ }^{\mathrm{TM}}$ vaccination: review of the global data, 2006. Vaccine 25, 3085-3089.

CLSI (2009). Performance Standards for Antimicrobial Susceptibility Testing, 19th Informational Supplement. CLSI document M100-S19. Wayne, PA: Clinical and Laboratory Standards Institute.
Enright, M. C. \& Spratt, B. G. (1998). A multilocus sequence typing scheme for Streptococcus pneumoniae: identification of clones associated with serious invasive disease. Microbiology 144, 30493060.

Feil, E. J., Li, B. C., Aanensen, D. M., Hanage, W. P. \& Spratt, B. G. (2004). eBURST: inferring patterns of evolutionary descent among clusters of related bacterial genotypes from multilocus sequence typing data. J Bacteriol 186, 1518-1530.

Hanage, W. P. (2008). Serotype-specific problems associated with pneumococcal conjugate vaccination. Future Microbiol 3, 23-30.

Hanage, W. P., Kaijalainen, T. H., Syrjänen, R. K., Auranen, K., Leinonen, M., Mäkelä, P. H. \& Spratt, B. G. (2005). Invasiveness of serotypes and clones of Streptococcus pneumoniae among children in Finland. Infect Immun 73, 431-435.

Hausdorff, W. P., Bryant, J., Paradiso, P. R. \& Siber, G. R. (2000). Which pneumococcal serogroups cause the most invasive disease: implications for conjugate vaccine formulation and use, part I. Clin Infect Dis 30, 100-121.

Hava, D. L., LeMieux, J. \& Camilli, A. (2003). From nose to lung: the regulation behind Streptococcus pneumoniae virulence factors. Mol Microbiol 50, 1103-1110.

Marrie, T. J. (2000). Community-acquired pneumonia in the elderly. Clin Infect Dis 31, 1066-1078.

McGee, L., McDougal, L., Zhou, J., Spratt, B. G., Tenover, F. C., George, R., Hakenbeck, R., Hryniewicz, W., Lefévre, J. C. \& other authors (2001). Nomenclature of major antimicrobial-resistant clones of Streptococcus pneumoniae defined by the pneumococcal molecular epidemiology network. J Clin Microbiol 39, 25652571.

Motlova, J., Benes, C. \& Kriz, P. (2009). Incidence of invasive pneumococcal disease in the Czech Republic and serotype coverage by vaccines, 1997-2006. Epidemiol Infect 137, 562-569.

Sandgren, A., Sjostrom, K., Olsson-Liljequist, B., Christensson, B., Samuelsson, A., Kronvall, G. \& Henriques Normark, B. (2004). Effect of clonal and serotype-specific properties on the invasive capacity of Streptococcus pneumoniae. J Infect Dis 189, 785-796.

Sorensen, U. B. (1993). Typing of pneumococci by using 12 pooled antisera. J Clin Microbiol 31, 2097-2100.

Takala, A. K., Vuopio-Varkila, J., Tarkka, E., Leinonen, M. \& Musser, J. M. (1996). Subtyping of common pediatric pneumococcal serotypes from invasive disease and pharyngeal carriage in Finland. J Infect Dis 173, 128-135.

Watson, D. A. \& Musher, D. M. (1999). A brief history of the pneumococcus in biomedical research. Semin Respir Infect 14, 198208.

Zemlickova, H., Urbaskova, P., Adamkova, V., Motlova, J., Lebedova, V. \& Prochazka, B. (2006). Characteristics of Streptococcus pneumoniae, Haemophilus influenzae, Moraxella catarrhalis and Staphylococcus aureus isolated from the nasopharynx of healthy children attending day-care centres in the Czech Republic. Epidemiol Infect 134, 11791187. 